\title{
The Determination of the Optimal Material Proportion in Natural Fiber-Cement Composites Using Design of Mixture Experiments
}

\author{
Chuckaphun Aramphongphun ${ }^{1, a}$ *, Kampanart Ungtawondee ${ }^{2, b}$ \\ and Duangrudee Chaysuwan ${ }^{3, \mathrm{c}}$ \\ ${ }^{1}$ Department of Industrial Engineering, Faculty of Engineering, Kasetsart University, Chatuchak, \\ Bangkok 10900, Thailand \\ ${ }^{2}$ Graduate Program in Engineering Management, Department of Industrial Engineering, Faculty of \\ Engineering, Kasetsart University, Chatuchak, Bangkok 10900, Thailand \\ ${ }^{3}$ Department of Materials Engineering, Faculty of Engineering, Kasetsart University, Chatuchak, \\ Bangkok 10900, Thailand \\ âfengchar@ku.ac.th1 ${ }^{1}{ }^{b}$ ungkampanart@gmail.com, ${ }^{c}$ fengddc@ku.ac.th
}

\begin{abstract}
This research aims to determine the optimal material proportion in a natural fiber-cement composite as an alternative to an asbestos fibercement composite while the materials cost is minimized and the properties still comply with Thai Industrial Standard (TIS) for applications of profile sheet roof tiles. Two experimental sets were studied in this research. First, a three-component mixture of (i) virgin natural fiber, (ii) synthetic fiber and (iii) cement was studied while the proportion of calcium carbonate was kept constant. Second, an additional material, recycled natural fiber from recycled paper, was used in the mixture. The four-component mixture was then studied. Constrained mixture design was applied to design the two experimental sets above. The experimental data were then analyzed to build the mixture model. In addition, the cost of each material was used to build the materials cost model. These two mathematical models were then employed to optimize the material proportion of the natural fiber-cement composites. In the three-component mixture, it was found that the optimal material proportion was as follows: $3.14 \%$ virgin natural fiber, $1.20 \%$ synthetic fiber and $75.67 \%$ cement while the materials cost was reduced by $12 \%$. In the four-component mixture, it was found that the optimal material proportion was as follows: $3.00 \%$ virgin natural fiber, $0.50 \%$ recycled natural fiber, $1.08 \%$ synthetic fiber, and $75.42 \%$ cement. The materials cost was reduced by $14 \%$. The confirmation runs of 30 experiments were also analyzed statistically to verify the results.
\end{abstract}

\section{Introduction}

Currently, the number of living residences worldwide has expanded continuously. The roof tile is one of the essential components in residential construction. The roof tile is

* Corresponding author:fengchar@ku.ac.th 
commonly made of fiber-cement composites in the form of profile sheets. In the industry, fiber-cement composites for the profile sheet roof tiles are available in two main types as follows.

(i) Asbestos type fiber-cement composites, which mainly consist of asbestos fiber and cement.

(ii) Non-asbestos type fiber-cement composites, which mainly consist of natural fiber (e.g. cellulose fiber), synthetic fiber (e.g. polyester, polyvinyl alcohol or PVA) and cement. In this type of fiber-cement composites, natural and synthetic fibers are used instead of asbestos fiber.

Asbestos is a generic name of fibrous mineral silicates. It has several outstanding properties including high toughness, tensile strength, heat resistance, and chemical resistance. Although asbestos has several advantages, it can cause serious diseases in human lungs. Therefore, other materials, including natural and synthetic fibers, have been used to replace asbestos fiber. The cost and proportion of all materials in both the asbestos and the non-asbestos fiber-cement composites for the profile sheet roof tiles are summarized in Table 1 .

Table 1. cost and proportion of all materials in both the asbestos and the non-asbestos fiber-cement composites for profile sheet roof tiles.

\begin{tabular}{|c|c|c|c|}
\hline Materials & $\begin{array}{c}\text { Cost } \\
\text { (Baht per ton) }\end{array}$ & $\begin{array}{l}\text { Asbestos Fiber- } \\
\text { Cement } \\
\text { Proportion (\%) }\end{array}$ & $\begin{array}{c}\text { Non-asbestos } \\
\text { Fiber-Cement } \\
\text { Proportion (\%) }\end{array}$ \\
\hline 1. Type 1 Cement & 2,040 & 90 & 74.5 \\
\hline 2. Asbestos fiber & 14,500 & 10 & -- \\
\hline 3. Natural fiber & 25,310 & -- & 4.0 \\
\hline 4. Synthetic fiber & 104,290 & -- & 1.5 \\
\hline 5. Calcium Carbonate & 980 & -- & 20.0 \\
\hline Total cost (Baht per ton) & & 3,286 & 4,292 \\
\hline
\end{tabular}

Note: Currency exchange rate: 36 Baht $\sim 1$ US Dollar on December 2015

According to Table 1, it was found that the cost of non-asbestos fiber-cement composite is approximately $25 \%$ higher than that of asbestos fiber-cement composite. To increase competitive opportunities in the market, the cost of the non-asbestos fiber-cement composite should be reduced. Therefore, this research aims to determine the optimal proportion of materials in the non-asbestos fiber-cement composite to reduce the materials cost using mixture design, and that their properties still meet Thai Industrial Standard for applications of profile sheet roof tiles. Virgin and recycled natural fibers and synthetic fibers were used to replace asbestos fiber in the fiber-cement composite. The proportion of main materials in the non-asbestos fiber-cement composite was optimized to reduce the materials cost. These main materials included (i) natural fiber such as cellulose fiber, (ii) synthetic fiber such as PVA fiber, and (iii) type 1 cement. Due to the high cost of natural and synthetic fibers, the main effort focused on reducing the proportion of these two fiber types as much as possible. 


\section{Related Research and Theory}

Savastano et al. [1] designed the experiments to study the effect of the amount of cellulose fiber on the modulus of rupture and toughness by varying the amount of fiber at $4 \%, 8 \%$, and $12 \%$. These two properties of the products at a life of 28 and 42 days were also studied. The results showed that both the modulus of rupture and toughness were proportional to the amount of cellulose fiber that was used to reinforce. In addition, it was found that the modulus of rupture with a product life of 28 and 42 days was not different while toughness at the product life of 42 days was lower than that with a product life of 28 days. Bezerra et al. [2] designed an experimental mixture by adjusting the amount of synthetic fiber, Polyvinyl Alcohol (PVA), at 1\%, 2\%, and 3\% to study the effect of the amount of synthetic fiber on the modulus of rupture and toughness. It was found that the amount of synthetic fiber had more significance than other components.

Moslemi [3] compared the properties of different types of fiber that were used to manufacture fiber cement products using the Hatschek process. It was found that the strongest fibers were Kevlar, carbon, and wood pulp, respectively. However, the cost of the Kevlar and carbon fibers was relatively high, and they were consequently not suitable for use in production. Therefore, the most suitable type of fiber used for the fiber cement products was wood pulp. Khorami and Ganjian [4] designed mixture experiments using recycled waste cardboard and limestone powder to reduce the materials cost of fibercement products. In addition, the effects of the main components in the fiber-cement material on the strength of the product were studied. These included the amount of cellulose pulp from waste cardboard and the amount of limestone powder in the fiber-cement material. The optimal proportions of cellulose pulp from waste cardboard and limestone powder in the fiber-cement material were also determined. It was found that the optimal proportions that provided the greatest strength for the fiber-cement material were as follows: cellulose pulp from waste cardboard at $8 \%$ and limestone powder at $10 \%$.

A mixture design is one of the design of experiment techniques used to find the suitable proportions of two or more components in a mixture. The sum of all component proportions must always be 1.0 (or 100\%). Therefore, when the proportion of a given component increases, the proportions of the other components in the mixture decrease in the same corresponding ratio. A mixture design is used when the responses, such as mechanical properties, mainly depend on the proportion of the components in the mixture. This can be expressed as shown in Eq. 1.

$$
\sum_{i=1}^{q} x_{i=x_{1}+x_{2}+\cdots+x_{q}=1.0}
$$

where $q$ is total number of components in the mixture. $x_{i}$ is a proportion of component $i$ in the mixture.

Because the proportions of all components significantly affect the response and must sum to 1 , a regression model of the mixture is then built without a constant $\left(\beta_{0}\right)$ in the model. The regression model of the mixture design was expressed as shown in Eq. 2. 


$$
\widehat{y}=\sum_{i=1}^{k} \widehat{\beta}_{\iota} \chi_{i}+\sum \sum_{i<j}^{k} \widehat{\beta_{\iota \jmath}} \chi_{i} \chi_{j}
$$

where $\hat{y}$ is an estimated response variable in the regression model. $\widehat{\beta}_{l}$ is an estimated coefficient of the independent variable $i . \widehat{\beta_{l j}}$ is an estimated coefficient of interaction term of independent variables $i j . x_{i}$ is an independent variable $i . x_{i} x_{j}$ is an interaction term of independent variables $i j . k$ is total number of components in the mixture.

\section{Description and Results of Mixture Experiments}

In the three-component mixture, the proportion of natural fiber was varied between $2.5 \%-3.5 \%$, the proportion of synthetic fiber was varied between $1.0 \%-1.5 \%$, and the proportion of cement was not lower than $75 \%$ while the proportion of calcium carbonate remained constant at $20 \%$. These ranges of the component proportions were selected to avoid any other defects in the fiber-cement composite. In the four-component mixture, the proportion of natural fiber was varied between $2 \%-3 \%$, the proportion of natural fiber from recycled cardboard was varied between $0.5 \%-1.5 \%$, the proportion of synthetic fiber was varied between $1.0 \%-1.5 \%$, and the proportion of cement was not lower than $75 \%$ while the proportion of calcium carbonate remained constant at $20 \%$. These ranges of the component proportions were selected to avoid any other defects in the fiber-cement composite. Mixture experiments were performed under the same conditions, the same parameters, the same machine, and the same batch of materials. Samples of the fiber-cement composites in the form of profile sheet were collected to test product quality according to the industrial standard. These tests include: (i) fracture resistance test and (ii) heat-rain resistance test. The fracture resistance should not be less than $1,500 \mathrm{~N}$ and the heat-rain resistance test should not be less than 25 cycles. Moreover, the fiber-cement composite also passed the leak and impact resistance tests. Mixture design was applied to plan the experiments with the fiber-cement composites. The responses in the experiments included: (i) materials cost, (ii) fracture load resistance, and (iii) heat-rain resistance while the products are also required to pass the leak and impact resistance tests.

The Three-component Mixture of Virgin Natural Fiber, Synthetic Fiber, and Cement. According to Table 1, the cost model of the materials was built as shown in Eq. 3 .

$$
\text { Materials cost }=2,040 * X_{1}+25,310 * X_{2}+104,290 * X_{3} \text {. }
$$

where $X_{1}, X_{2}$, and $X_{3}$ are the proportions of cement, virgin natural fiber, and synthetic fiber in the fiber-cement composite, respectively.

The regression analysis of fracture load resistance was performed by using Minitab. The analysis of the full model, including all main components and interaction terms, was first analyzed. It was found that all the interaction terms were not significant ( $p$-value $>$ 0.05). Therefore, all the interaction terms were removed and the reduced model was then analyzed. The regression model of fracture load resistance was obtained and summarized as shown in Eq. 4.

$$
\text { Fracture load resistance }=330.5 * X_{1}+23,772.6 * X_{2}+52,759.2 * X_{3} \text {. }
$$


where $X_{1}, X_{2}$, and $X_{3}$ are the proportions of cement, virgin natural fiber, and synthetic fiber in the fiber-cement composite, respectively.

The regression analysis of heat-rain resistance (HRT) was performed by using Minitab. The analysis of the full model, including all main components and interaction terms, was first analyzed. It was found that all the interaction terms were not significant ( $p$-value $>$ $0.05)$. Therefore, all the interaction terms were removed and the reduced model was then analyzed. The reduced regression model of heat-rain resistance (HRT) was obtained and summarized as shown in Eq. 5.

$$
\text { Heat-rain resistance }=-7.35 * X_{1}+381.22 * X_{2}+1,135.51 * X_{3}
$$

where $X_{1}, X_{2}$, and $X_{3}$ are the proportions of cement, virgin natural fiber, and synthetic fiber in the fiber-cement composite, respectively.

The four-component mixture of cement, virgin natural fiber, recycled natural fiber, and synthetic fiber. According to Table 1, the cost model of the materials was built as shown in Eq. 6.

Materials cost $=2,040 * X_{1}+25,310 * X_{2}+104,290 * X_{3}+15,050 * X_{4}$

where $X_{1}, X_{2}, X_{3}$ and $X_{4}$ are the proportions of cement, virgin natural fiber, synthetic fiber, and recycled natural fiber in the fiber-cement composite, respectively.

The regression analysis of the fracture load resistance was performed by using Minitab. The analysis of the full model, including all main components and interaction terms, was first analyzed. It was found that some interaction terms were not significant ( $p$-value $>$ 0.05 ). Therefore, the non-significant interaction terms were removed and the reduced model was then analyzed. The reduced regression model of fracture load resistance was obtained and summarized as shown in Eq. 7.

$$
\begin{array}{r}
\text { Fracture load resistance }=533 * X_{1}+54,560 * X_{2}+6,333,260 * X_{3}+45,950 * X_{4} \\
-6,407,559 * X_{1} X_{3}-8,105,279 * X_{2} X_{3}-7,943,509 * X_{3} X_{4}
\end{array}
$$

where $X_{1}, X_{2}, X_{3}$, and $X_{4}$ are the proportions of cement, virgin natural fiber, synthetic fiber, and recycled natural fiber in the fiber-cement composite, respectively.

The regression analysis of heat-rain resistance (HRT) was performed by using Minitab. The analysis of the full model, including all main components and interaction terms, was first analyzed. It was found that all the interaction terms were not significant ( $p$-value $>$ 0.05 ). Therefore, all the interaction terms were removed and the reduced model was then analyzed. The reduced regression model of heat-rain resistance (HRT) was obtained and summarized as shown in Eq. 8.

$$
\begin{aligned}
\text { Heat-rain resistance }=-37 * & X_{1}+401 * X_{2}-120,711 * X_{3}+306 * X_{4} \\
& +125,724 * X_{1} X_{3}+123,286 * X_{2} X_{3}+115,972 * X_{3} X_{4}
\end{aligned}
$$

where $X_{1}, X_{2}, X_{3}$, and $X_{4}$ are the proportions of cement, virgin natural fiber, synthetic fiber, and recycled natural fiber in the fiber-cement composite, respectively. 


\section{Response Optimization}

In this research, the goals of the response optimization were to minimize the materials cost to be lower than 3,667 baht per ton (reduced by 10\%), to maximize the fracture load resistance to be greater than $1,500 \mathrm{~N}$ and to target the heat-rain resistance to be 25 cycles. The three responses, including (i) materials cost, (ii) fracture load resistance, and (iii) heatrain resistance, were optimized by using the desirability function. The corresponding regression models of the materials cost, fracture load resistance, and heat-rain resistance were employed to obtain the optimal responses. The results of the response optimization using Minitab for the three-component mixture and four-component mixture are shown in Fig. 1a and Fig. 1b, respectively.

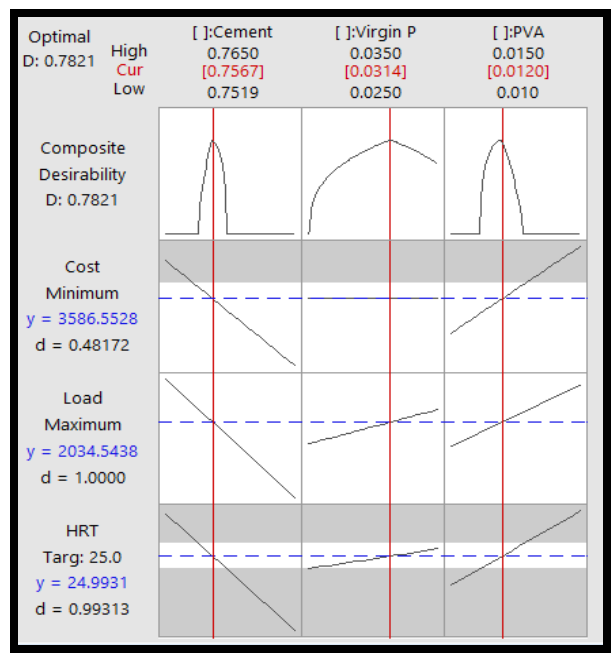

(a)

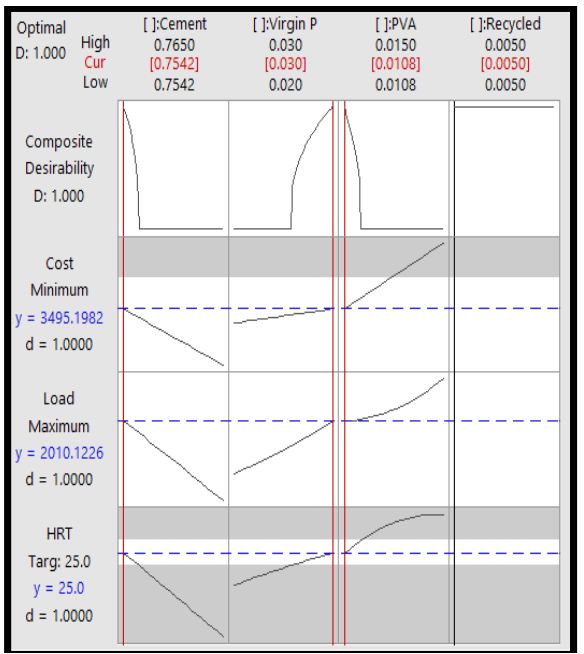

(b)

Figure 1. The optimization plots from Minitab used to determine the optimal material proportions in (a) the three-component mixture and (b) the four-component mixture.

Confirmation runs of 30 experiments were then performed to verify the optimal responses in both the three-component and four-component mixtures.

\section{Conclusions}

Design and analysis of the mixture experiments for the fiber-cement composites were performed to reduce the materials cost while their properties still followed the industrial standard for applications of profile sheet roof tiles. The materials cost model and the mixture model of the fracture load resistance and the heat-rain resistance were built and then employed to determine the optimal material proportions in the mixture as summarized in Table 2. For the three-component mixture, the proportion of cement at $75.67 \%$, the proportion of virgin natural fiber at $3.14 \%$, and the proportion of synthetic fiber at $1.20 \%$ were obtained to achieve the optimal responses, which were at the minimum materials cost of 3,586.55 baht per ton, the maximum fracture load resistance of 2,034.54 $\mathrm{N}$ and the target heat-rain resistance of 25 cycles. By including the cost of calcium carbonate (196 baht per ton), the total materials cost of the three-component mixture became 3,782.55 $(=3,586.55+$ 196) baht per ton. As shown in Table 1, the materials cost of the three-component mixture could be reduced from the current materials cost of 4,292 to 3,782.55 baht per ton (or 
reduced by $12 \%$ ). For the four-component mixture, the proportion of cement at $75.42 \%$, the proportion of virgin natural fiber at $3.00 \%$, the proportion of synthetic fiber at $1.08 \%$ and the proportion of recycled natural fiber of $0.5 \%$ were obtained to achieve the optimal responses, which were at the minimum materials cost of $3,495.19$ baht per ton, the maximum fracture load resistance of 2,010.12 $\mathrm{N}$ and the target heat-rain resistance of 25 cycles. By including the cost of calcium carbonate (196 baht per ton), the total materials cost of the four-component mixture became 3,691.19 $(=3,495.19+196)$ baht per ton. According to Table 1, the materials cost of the four-component mixture could be reduced from the current materials cost of 4,292 to 3,691.19 baht per ton (or reduced by $14 \%$ ).

Table 2. A Summary Table Of The Optimal Component Proportions And Materials Cost In The Mixture Experiments

\begin{tabular}{|c|c|c|c|c|}
\hline & & \multicolumn{3}{|c|}{ Composites } \\
\hline Materials Proportion and Cost & $\begin{array}{l}\text { Asbestos } \\
\text { Fiber- } \\
\text { Cement } \\
\text { Composite }\end{array}$ & $\begin{array}{c}\text { Current } \\
\text { Proportion }\end{array}$ & $\begin{array}{c}\text { Three- } \\
\text { Component } \\
\text { Mixture }\end{array}$ & $\begin{array}{c}\text { Four- } \\
\text { Component } \\
\text { Mixture } \\
\end{array}$ \\
\hline 1. Type 1 Cement (\%) & 90 & 74.50 & 75.67 & 75.42 \\
\hline 2. Virgin Natural Fiber (\%) & -- & 4.00 & 3.14 & 3.00 \\
\hline 3. Synthetic Fiber (\%) & -- & 1.50 & 1.20 & 1.08 \\
\hline 4. Recycled Natural Fiber (\%) & -- & -- & -- & 0.50 \\
\hline 5. Calcium Carbonate (\%) & -- & 20.00 & 20.00 & 20.00 \\
\hline 6. Asbestos Fiber (\%) & 10 & -- & -- & -- \\
\hline $\begin{array}{l}\text { Total Materials Cost (Baht per } \\
\text { ton) }\end{array}$ & 3,286 & 4,292 & $3,782.55$ & $3,691.19$ \\
\hline$\%$ Materials Cost Reduction & & & 12 & 14 \\
\hline
\end{tabular}

Note: Currency exchange rate: 36 Baht $\sim 1$ US Dollar on December 2015

\section{References}

1. H. Savastano Jr, P.G. Warden and R.S.P. Coutts, Brazilian waste fibres as reinforcement for cement-based composites, Cement \& Concrete Composites, 22(5) (2000) 379-384.

2. E.M. Bezerra, A.P. Joaquim, H. Savastano Jr, V.M. John and V. Agopyan, The effect of different mineral additions and synthetic fiber contents on properties of cement based composites, Cement \& Concrete Composites, 28(6) (2006) 555-563.

3. A. Moslemi, Technology and market considerations for fiber cement, Fiber Composites Conference, 5 (2005) 113-129.

4. M. Khorami and E. Ganjian, The effect of limestone powder, silica fume and fibre content on flexural behaviour of cement composite reinforced by waste Kraft pulp, Construction and Building Materials, 46 (2013) 142-149. 